\title{
2.1-W picosecond passively mode-locked external-cavity semiconductor laser
}

\author{
A. Aschwanden, D. Lorenser, H. J. Unold, and R. Paschotta \\ Department of Physics, Institute of Quantum Electronics, Swiss Federal Institute of Technology (ETH), ETH Zürich Hönggerberg, \\ Wolfgang-Pauli-Strasse 16, 8093 Zürich, Switzerland \\ E. Gini \\ Frontiers in Research: Space and Time (FIRST) Center for Micro- and Nanoscience, Swiss Federal Institute of Technology (ETH), \\ ETH Zürich Hönggerberg, Wolfgang-Pauli-Strasse 10, 8093 Zürich, Switzerland \\ U. Keller \\ Department of Physics, Institute of Quantum Electronics, Swiss Federal Institute of Technology (ETH), ETH Zürich Hönggerberg, \\ Wolfgang-Pauli-Strasse 16, 8093 Zürich, Switzerland
}

Received August 25, 2004

\begin{abstract}
We demonstrate an optically pumped passively mode-locked external-cavity semiconductor laser generating 4.7-ps pulses at $957 \mathrm{~nm}$ with as much as $2.1 \mathrm{~W}$ of average output power and a 4-GHz repetition rate. Compared with earlier results, the chirp of the pulses has been greatly reduced by use of an intracavity etalon. Apart from restricting the bandwidth, the etalon also helps optimize wavelength-dependent gain parameters and dispersion. (C) 2005 Optical Society of America
\end{abstract}

OCIS codes: $140.4050,140.5960,140.7090$.

Mode-locked lasers generating multigigahertz picosecond pulse trains with multiwatt average power are suitable for a wide variety of applications, such as optical clocking, external-cavity frequency doubling, and telecommunication. For example, a $10-\mathrm{GHz}$ $\mathrm{Nd}: \mathrm{YVO}_{4}$ laser with $2.1 \mathrm{~W}$ of output has been used to synchronously pump an optical parametric oscillator ${ }^{1}$ whose signal wavelength range covered the $\mathrm{S}, \mathrm{C}$, and $\mathrm{L}$ telecommunication bands.

To overcome the serious power limitations of mode-locked edge-emitting semiconductor lasers, we demonstrated $^{2}$ an optically pumped vertical externalcavity surface-emitting laser (VECSEL) combined with a semiconductor saturable absorber mirror ${ }^{3,4}$ (SESAM) for passive mode locking. Since the first demonstration, ${ }^{2}$ we have been able to report high average output powers of $1 \mathrm{~W}$ in 15-ps pulses at a $6-\mathrm{GHz}$ repetition rate $^{5}$ and $1.9 \mathrm{~W}$ in 27 -ps pulses at a $1.5-\mathrm{GHz}$ repetition rate. ${ }^{6}$ Here we demonstrate a passively mode-locked VECSEL with an even-higher output power of $2.1 \mathrm{~W}$ with a significantly reduced pulse duration of only $4.7 \mathrm{ps}$ and drastically reduced chirp. Thus passively mode-locked VECSELs have now reached a performance level that is somewhat better than that of the more-traditional passively mode-locked diode-pumped solid-state lasers when optimized for high output power at a multigigahertz pulse repetition rate. ${ }^{7,8}$ In addition, mode-locked VECSELs also offer the ability of operation at different laser wavelengths and the potential for cheap mass production with wafer-scale integration. An important aspect for passive mode locking is the small gain saturation fluence of semiconductor materials, which greatly helps suppress $Q$-switching instabilities even at multigigahertz repetition rates., ${ }^{9,10}$ Furthermore, their broad amplification bandwidth allows the generation of sub-500-fs pulses. ${ }^{11,12}$

The gain structure of our device consists of three parts: a highly reflecting bottom mirror, the active region, and an antireflective section. The $\mathrm{AlAs} / \mathrm{Al}_{0.2} \mathrm{Ga}_{0.8} \mathrm{As}$ bottom mirror is optimized for high reflectivity at the pump $(808 \mathrm{~nm})$ and laser wavelength $(\approx 960 \mathrm{~nm})$ for an incident angle of $45^{\circ}$ and normal incidence, respectively. Most of the pump light is absorbed in the active region, which contains seven $\mathrm{In}_{0.13} \mathrm{Ga}_{0.87}$ As quantum wells (QWs) placed in the antinodes of the standing-wave pattern. The QWs have $\mathrm{GaAs}_{0.94} \mathrm{P}_{0.06}$ strain-compensating layers on both sides and are separated by pump-absorbing GaAs sections. The residual reflectivity of the top $\mathrm{AlAs} / \mathrm{Al}_{0.2} \mathrm{Ga}_{0.8} \mathrm{As}$ section is $<1 \%$ at the laser wavelength. Together with the bottom mirror, it forms a Gires-Tournois interferometer. We designed our device for positive group-delay dispersion (GDD) by adjusting the total optical thickness of the active region. However, because of growth errors, the transition wavelength from positive to negative GDD was shifted from 958 to $954 \mathrm{~nm}$, leading to negative GDD at the wavelength of maximum gain. A similar but much weaker Gires-Tournois interferometer effect occurs on the SESAM. As discussed below, positive GDD is desirable for the generation of nearly transform-limited pulses. ${ }^{13}$

The gain structure was grown in reverse order by metal-organic vapor-phase epitaxy and soldered to a copper heat sink. The GaAs substrate was then removed by wet etching, yielding a structure with a very low thermal impedance, allowing high output powers 
without excessive heating. By use of the analytical expressions given in Ref. 5, the thermal impedance was calculated to be approximately $8 \mathrm{~K} / \mathrm{W}$ for a pump spot radius of $175 \mu \mathrm{m}$ and a semiconductor structure thickness of $6 \mu \mathrm{m}$. Details on the growth and processing can be found in Ref. 5 .

In the $V$-shaped laser cavity (Fig. 1) the gain structure serves as a folding mirror. It is pumped on a spot with an $\approx 175-\mu \mathrm{m}$ radius by use of a fiber-coupled laser diode module. The cavity end mirrors are the SESAM and the output coupler with a $38-\mathrm{mm}$ curvature radius and $2.5 \%$ transmission. The SESAM contains an 8.5-nm-thick $\operatorname{In}_{0.15} \mathrm{Ga}_{0.85} \mathrm{As}$ QW grown with molecular beam epitaxy at a low temperature. It shows an exciton around $959 \mathrm{~nm}$ and has a modulation depth of approximately $1 \%$. An $\approx 20$ - $\mu \mathrm{m}$-thick uncoated fused-silica etalon is inserted near the SESAM.

The circulating pulse of a mode-locked VECSEL saturates the gain and the absorber, changing the carrier densities and therefore the refractive indices. The resulting nonlinear phase changes $\Delta \varphi_{g}(t)$ in the gain medium and $\Delta \varphi_{a}(t)$ in the absorber are proportional to the time-dependent gain $g(t)$ and loss $q(t)$, respectively, and to the corresponding linewidth enhancement factors ${ }^{14,15} \alpha_{g}$ and $\alpha_{a}$. They are responsible for the strong chirp that is often observed in mode-locked semiconductor lasers, in particular for negative intracavity GDD. However, it has been found $^{13}$ that, with positive GDD, stable and nearly transform-limited pulses can be formed because the nonlinear and dispersive phase changes cancel each other.

In our laser setup the total intracavity GDD is dominated by that of the gain structure (Fig. 2 on the bottom in gray) and of the etalon, whereas that of the SESAM is comparatively small. The top graph of Fig. 2 illustrates the transmission and the GDD of a $20-\mu \mathrm{m}$-thick uncoated fused-silica etalon when it is angle tuned to $960 \mathrm{~nm}$. At the points of maximum transmission the GDD is zero, but it can reach $\pm 1200 \mathrm{fs}^{2}$ at the slopes of the transmission curve. The bottom graph of Fig. 2 shows the sum of the GDD contributions from the gain structure and the etalon for etalon angles of $10^{\circ}, 13.5^{\circ}$, and $17^{\circ}$, demonstrating that the etalon not only determines the emission wavelength but also significantly influences the intracavity GDD. The chirp-related ${ }^{13}$ spectral shifts make it difficult to predict how much the laser wavelength will differ from the wavelength of maximum etalon transmission and thus how much GDD the etalon will contribute. Even the sign of the etalon GDD cannot be easily determined.

In experiments with a similar laser setup we observed a relatively complicated dependence of the achieved pulse duration on the laser wavelength when tuning the wavelength by rotating the etalon. Angular positions with stable mode locking alternate with unstable states, and small wavelength changes can lead to large changes in pulse duration and output power. We tried to understand this kind of behavior with numerical simulations similar to those in Ref. 13, but this turned out to be difficult for a number of reasons. One problem arises from the significant uncertainties in the etalon GDD and other parameters, such as the linewidth enhancement factors. In addition, we suspect that the temperature difference between the center and the outer parts of the pumped region introduces significant inhomogeneous broadening that cannot be implemented easily in a model.

Despite these difficulties, however, we experimentally found situations leading to favorable performance. We optimized the output power and pulse duration of the laser by varying the etalon angle and heat-sink temperature of the gain medium. We achieved best performance with $2.1 \mathrm{~W}$ of average output power in a $4-\mathrm{GHz}$ pulse train with $18.9 \mathrm{~W}$ of pump power and a heat-sink temperature of $-4{ }^{\circ} \mathrm{C}$. The etalon angle was close to normal incidence. Since the exact etalon thickness is not known, it is not possible to make a statement about the intracavity GDD

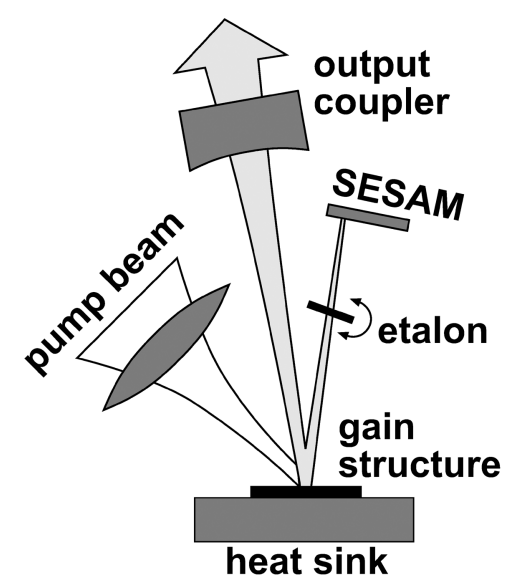

Fig. 1. Cavity setup for the passively mode-locked VECSEL.
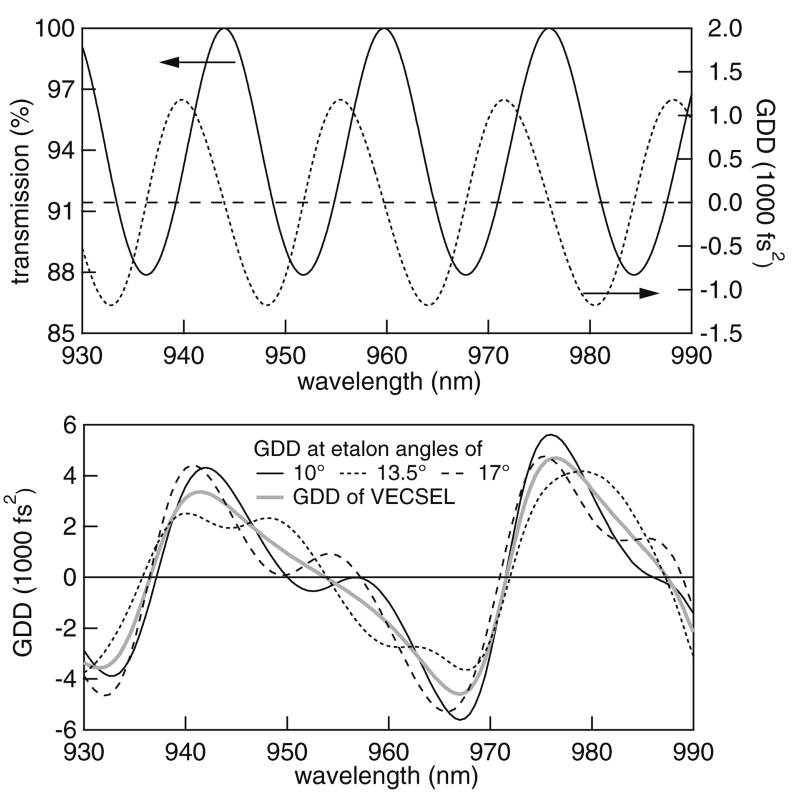

Fig. 2. Top, transmission and GDD of a 20- $\mu \mathrm{m}$-thick uncoated fused-silica etalon. Bottom, GDD of the gain structure (gray) and sum of the GDD of the gain structure and the etalon for different angular positions. 

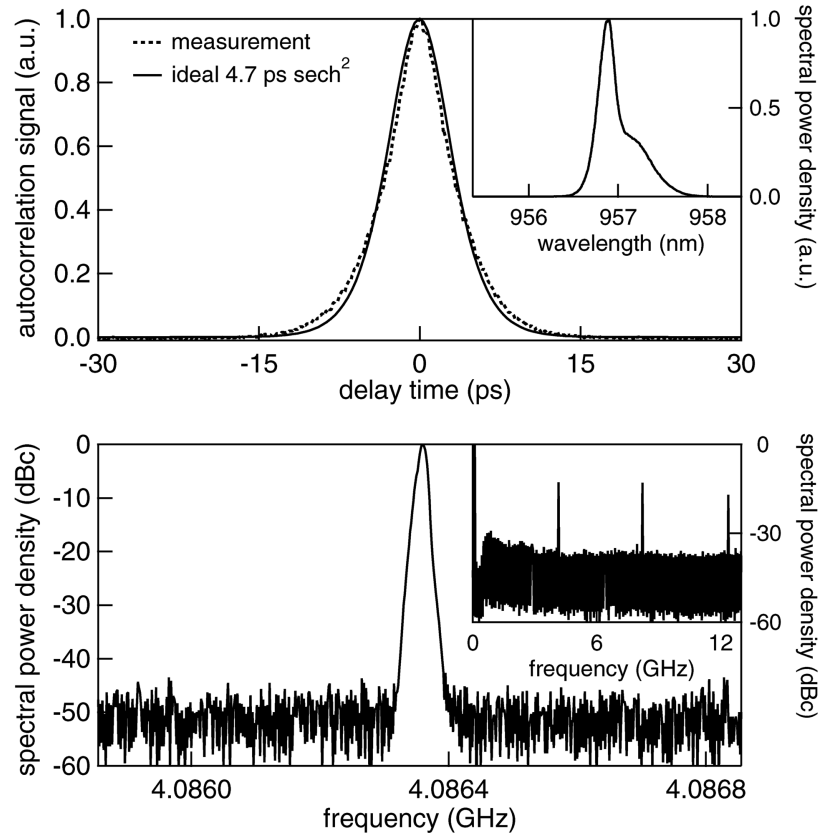

Fig. 3. Top, autocorrelation trace of the pulses with 4.7-ps duration. Inset, optical spectrum centered at $957 \mathrm{~nm}$. Bottom, rf spectrum of the pulse train at $4 \mathrm{GHz}$ with a $1-\mathrm{MHz}$ span and a $10-\mathrm{kHz}$ resolution bandwidth. Inset, measurement over a $12-\mathrm{GHz}$ span.

based on Fig. 2. The top graph of Fig. 3 shows the autocorrelation trace of the 4.7-ps pulses. Chirp-free pulses with the spectrum according to the inset of Fig. 3 would have approximately one half of this pulse duration. This deviation from the transform limit indicates imperfect compensation of the chirp, but it is far smaller than for previously demonstrated high-power mode-locked VECSELs, ${ }^{5,6}$ which provided pulses that were approximately 15-20 times greater than the transform limit. The $\mathrm{rf}$ spectrum of the signal from a fast photodiode, shown in Fig. 3 on the bottom, demonstrates stable mode locking at $4 \mathrm{GHz}$.

In conclusion, we have presented a passively mode-locked VECSEL with up to $2.1 \mathrm{~W}$ of average output power in 4.7-ps pulses at a $4-\mathrm{GHz}$ repetition rate. Compared with earlier results, the chirp of the output pulses has been greatly reduced by dispersion control with an etalon in the laser cavity. Although the details of the pulse formation appear to be rather complicated, the etalon was found to significantly improve the performance by optimizing the laser wavelength for best pulse shaping while also contributing GDD. For the first time to our knowledge, the performance of a mode-locked picosecond semiconductor laser has reached the level of passively mode-locked solid-state lasers optimized for high power in the multigigahertz regime. Given the rapid progress in high-power VECSELs, we expect that they could soon outperform all other mode-locked lasers in the multiwatt multigigahertz domain. Applications such as optical clocking could significantly profit from this development.

The authors are grateful for financial support from ETH Zürich and from an Intel-sponsored research agreement. A. Aschwanden's e-mail address is aschwanden@phys.ethz.ch.

\section{References}

1. S. Lecomte, R. Paschotta, M. Golling, D. Ebling, and U. Keller, J. Opt. Soc. Am. B 21, 844 (2004).

2. S. Hoogland, S. Dhanjal, A. C. Tropper, S. J. Roberts, R. Häring, R. Paschotta, and U. Keller, IEEE Photon. Technol. Lett. 12, 1135 (2000).

3. U. Keller, D. A. B. Miller, G. D. Boyd, T. H. Chiu, J. F. Ferguson, and M. T. Asom, Opt. Lett. 17, 505 (1992).

4. U. Keller, K. J. Weingarten, F. X. Kärtner, D. Kopf, B. Braun, I. D. Jung, R. Fluck, C. Hönninger, N. Matuschek, and J. Aus der Au, IEEE J. Sel. Top. Quantum Electron. 2, 435 (1996).

5. R. Häring, R. Paschotta, A. Aschwanden, E. Gini, F. Morier-Genoud, and U. Keller, IEEE J. Quantum Electron. 38, 1268 (2002).

6. A. Aschwanden, D. Lorenser, R. Häring, R. Paschotta, E. Gini, and U. Keller, in Conference on Lasers and Electro-Optics, Postconference Digest, Vol. 88 of OSA Trends in Optics and Photonics Series (Optical Society of America, Washington, D.C., 2003), paper CC1.

7. R. Paschotta, L. Krainer, S. Lecomte, G. J. Spühler, S. C. Zeller, A. Aschwanden, D. Lorenser, H. J. Unold, K. J. Weingarten, and U. Keller, "Picosecond pulse sources with multi-GHz repetition rates and high out-put power," New J. Phys. (to be published).

8. L. Krainer, R. Paschotta, S. Lecomte, M. Moser, K. J. Weingarten, and U. Keller, IEEE J. Quantum Electron. 38, 1331 (2002).

9. C. Hönninger, R. Paschotta, F. Morier-Genoud, M. Moser, and U. Keller, J. Opt. Soc. Am. B 16, 46 (1999).

10. R. Grange, M. Haiml, R. Paschotta, G. J. Spühler, L. Krainer, M. Golling, D. Ostinelli, and U. Keller, "New regime of inverse saturable absorption for selfstabilizing passively mode-locked lasers," Appl. Phys. B 1432-0649 (2004), published online.

11. A. Garnache, S. Hoogland, A. C. Tropper, I. Sagnes, G. Saint-Girons, and J. S. Roberts, Appl. Phys. Lett. 80, 3892 (2002)

12. S. Hoogland, A. C. Tropper, and J. S. Roberts, in Conference on Lasers and Electro-Optics, Postconference Digest, Vol. 88 of OSA Trends in Optics and Photonics Series (Optical Society of America, Washington, D.C., 2003), postdeadline paper.

13. R. Paschotta, R. Häring, U. Keller, A. Garnache, S. Hoogland, and A. C. Tropper, Appl. Phys. B 75, 445 (2002).

14. W. Jiang and J. Bowers, in Compact Sources of Ultrashort Pulses, I. I. N. Duling, ed. (Cambridge U. Press, New York, 1995), p. 208.

15. C. H. Henry, IEEE J. Quantum Electron. 18, 259 (1982). 